\title{
Locating Mobile Users Using Base Stations of Cellular Networks
}

\author{
Ilya M. Averin, Victor T. Ermolayev, Alexander G. Flaksman \\ Wireless Technologies Department, Mera NN, Russia \\ E-mail: ilya.averin@gmail.com, \{ave,ermol,flak\}@mera.ru \\ Received July 14, 2010; revised August 31, 2010; accepted September 29, 2010
}

\begin{abstract}
The location of mobile users served by cellular networks is considered. The chosen approach assumes that location is estimated through angular triangulation with three base stations (BSs). New estimates are proposed, which are described by closed-form expressions, and are alternatives to the maximum likelihood estimate. It is shown that the accuracy of the estimate coinciding with incircle center of the bearing triangle is close to the accuracy of the maximum likelihood estimate.
\end{abstract}

Keywords: Position Location, Location-based Services, Cellular Networks

\section{Introduction}

There is significant interest in location technologies in wireless networks. Information about the user position can be used for the development of location-based services [1] or for enhancing security [2]. The specifications of next generation cellular networks (LTE, WiMax) assume widespread implementation of spatial signal processing. This means that most BSs will be equipped with multiple antennas. Therefore, location techniques based on angular triangulation $[3,4]$ are of interest for advanced wireless networks. In [5], the angular triangulation approach applied to wireless networks is considered. It is shown that the corresponding location accuracy satisfies existing requirements [2]. However, only the somewhat complex maximum likelihood algorithm is presented there.

This paper proposes a positioning algorithm with low computational complexity and gives some estimates of its accuracy. The basic idea of the approach is to define a bearing triangle using the bearings of a mobile user measured at three BSs and then to try orthocenter, incircle center and centroid of this triangle as the candidate points of mobile user position.

The rest of the paper is organized as follows: the description of the proposed algorithm is given in Section 2, Section 3 discusses the simulation results and Conclusions are listed in Section 4.

\section{Positioning by Means of Angular Triangulation}

Figure 1 shows the geometry of angular triangulation with 3 BSs involved. The mobile is situated at the point $\left(x_{0}, y_{0}\right)$. True bearings referenced to the BSs are $\bar{\phi}_{1}, \bar{\phi}_{2}, \bar{\phi}_{3}$. The same figure depicts the situation when not true but the measured $\phi_{1}, \phi_{2}$ and $\phi_{3}$ bearings are known. Due to finite accuracy of direction finding, more than one cross point exists now and the intersections of bearing lines cause the appearance of bearing triangle $\Delta a b c$. This, in turn, makes the finding of the optimal estimate of the mobile's position non-trivial.

The bearing estimates can be introduced as independent Gaussian random values centered, of course, on the true bearings [6]. Therefore, the likelihood function with respect to the unknown exact angular directions $\bar{\phi}_{1}, \bar{\phi}_{2}, \bar{\phi}_{3}$ is written as

$$
P\left(\bar{\phi}_{1}, \bar{\phi}_{2}, \bar{\phi}_{3}\right)=\frac{1}{2 \pi \sqrt{2 \pi} \prod_{i=1}^{3} \sigma_{i}} \exp \left[-\sum_{i=1}^{3} \frac{\left(\phi_{i}-\bar{\phi}_{i}\right)^{2}}{2 \sigma_{i}^{2}}\right]
$$

where $\sigma_{i}$ is the standard deviation of the bearing error measured at the $\mathrm{BS}_{i}$.

In order to derive the maximum likelihood estimate of mobile user position, one should find the point where the likelihood function (1) reaches its extremum given three 


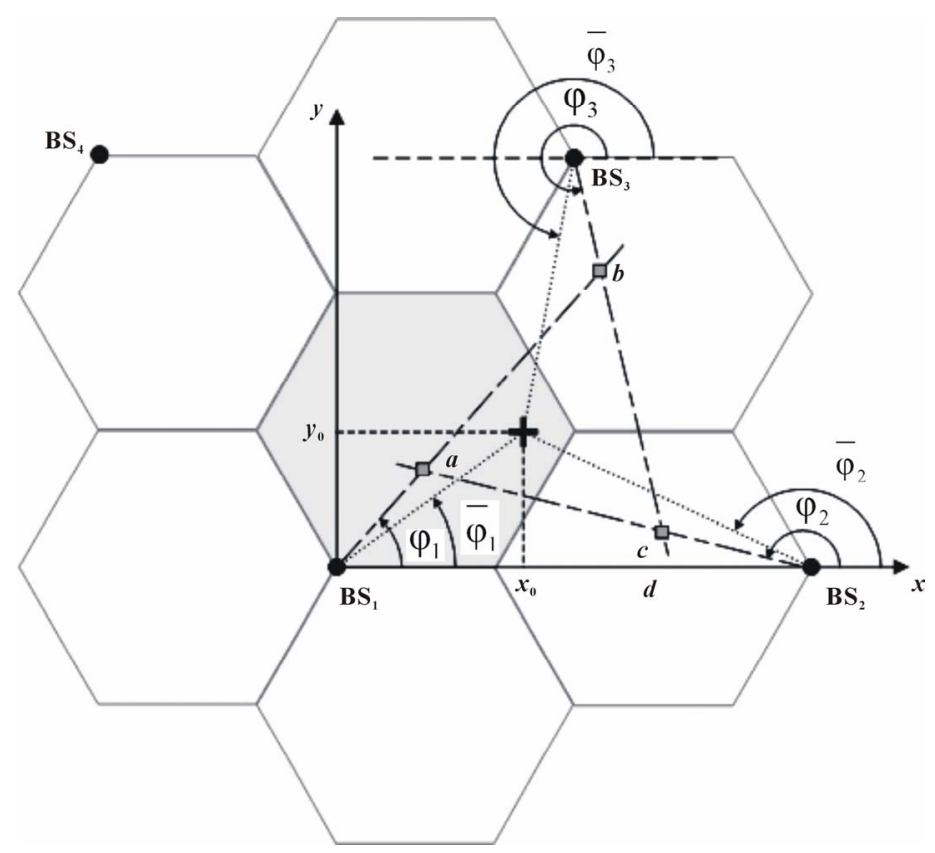

Figure 1. The geometry of angular triangulation with 3 BSs.

known bearing measurements $\phi_{1}, \phi_{2}$ and $\phi_{3}$. The solution of this problem gives the maximum likelihood estimate of bearings seen at the corresponding BSs. However, as depicted by Figure 1, the true angular $\bar{\phi}_{1}, \bar{\phi}_{2}, \bar{\phi}_{3}$ coordinates of the mobile are interdependent values. This makes the maximum likelihood approach quite complex from a computational point of view and an alternative way is of interest.

The bearing triangle $\Delta a b c$, like any other triangle, has such points as orthocenter, incircle center and centroid (the cross point of altitudes, angle bisectors and medians, respectively) [7]. If the positions of a triangle's vertices are known, it is straightforward to find coordinates of the points mentioned above using closed-form analytical expressions.

One can show, for example, that the coordinates of incircle center are described by the Equation (2) where the additional variables are introduced as Equation (3) and a, $\mathrm{b}, \mathrm{c},\left(x_{a}, y_{a}\right),\left(x_{b}, y_{b}\right),\left(x_{c}, y_{c}\right)$ are the triangle's side lengths and the coordinates of its vertices respectively.
Therefore, the coordinates of the orthocenter, incircle center and centroid are explicitly defined by the positions of the bearing triangle's vertices. In turn, they are fully dependant on three measured bearings. This allows us to consider the orthocenter, incircle center and centroid as simple-to-evaluate estimates of mobile user position. The next section addresses the issue of the positioning accuracy that can be attained with such an approach.

\section{Simulation Results}

To keep consistency with [5], we considered a network with hexagonal structure Figure 1 of four BSs $\left(\mathrm{BS}_{1}-\right.$ $\mathrm{BS}_{4}$ ). The structure shown corresponds to the "edge excited" network geometry, which assumes that a cell consists of three sectors and the BS is located at their vertices. Due to the properties of a hexagonal layout the distances between any neighboring BSs are the same and equal to $d$. Hereinafter, the coordinates normalized to $d$ are considered. This means that the distance between any

$$
\begin{gathered}
x_{I C}=\frac{\left(y_{b}-y_{a}\right)\left(x_{1}-x_{b}\right)\left(x_{2}-x_{a}\right)+\left(y_{2}-y_{A}\right)\left(x_{1}-x_{b}\right) x_{a}-\left(y_{1}-y_{b}\right)\left(x_{2}-x_{a}\right) x_{b}}{\left(y_{2}-y_{a}\right)\left(x_{1}-x_{b}\right)-\left(y_{1}-y_{b}\right)\left(x_{2}-x_{a}\right)} \\
y_{I C}=\frac{\left(x_{a}-x_{b}\right)\left(y_{1}-y_{b}\right)\left(y_{2}-y_{a}\right)+\left(y_{2}-y_{a}\right)\left(x_{1}-x_{b}\right) y_{b}-\left(y_{1}-y_{b}\right)\left(x_{2}-x_{a}\right) y_{a}}{\left(y_{2}-y_{a}\right)\left(x_{1}-x_{b}\right)-\left(y_{1}-y_{b}\right)\left(x_{2}-x_{a}\right)} \\
x_{1}=\frac{x_{a}+(a / c) x_{c}}{1+(a / c)}, \quad y_{1}=\frac{y_{a}+(a / c) y_{c}}{1+(a / c)}, \quad x_{2}=\frac{x_{c}+(b / a) x_{b}}{1+(b / a)}, \quad y_{1}=\frac{y_{c}+(b / a) y_{b}}{1+(b / a)}
\end{gathered}
$$


two nearest BSs is equal to 1 and any cell side has length $1 / 3$.

One assumption we made was about the placement of the mobile. It was assumed to be anywhere within the northeast sector of the central cell with uniform probability (this area is highlighted with the gray color in Figure 1.) Another assumption concerned the BSs involved in the location estimation process. Two scenarios were considered: the signal transmitted by the mobile was received by three BSs - either by $\mathrm{BS}_{1} \leftrightarrow \mathrm{BS}_{2} \leftrightarrow \mathrm{BS}_{3}$ (scenario 1) or by $\mathrm{BS}_{1} \leftrightarrow \mathrm{BS}_{2} \leftrightarrow \mathrm{BS}_{4}$ (scenario 2).

The task was to estimate the mobile's position based on the measured bearings. As position estimates are random values, the location accuracy was convenient to define by the CEPn metric. The CEP term stands for the Circular Error Probability and the parameter $n$ defines the required probability level. In other words, the CEPn metric is a radius of the circle comprising $n \%$ of the estimates obtained. The circle is centered about the true position of the located object. Greater CEPn for the fixed $n$ means worse location accuracy. In the case that normalized coordinates are used, the CEPn is a dimensionless value. In order to get the absolute value of positioning error one should multiply the obtained CEPn by the normalization factor (by BS-to-BS distance $d$ for the considered scenarios).

Simulations were performed to evaluate the location accuracy of the proposed algorithm. The corresponding results are shown in Figure 2, Figure 3 (for scenario 1) and Figure 4, Figure 5 (for scenario 2). The curves present the dependences of positioning error (in terms of the dimensionless CEPn) upon the standard deviation $\sigma$ of bearing error. The value of $\sigma$ was assumed to be the same for all BSs. For each value of $\sigma, 10^{5}$ random positions within the northeast sector of the central cell were considered. For every current position, three bearings were generated as Gaussian random values. This set of bearings was used to find the current estimate of mobile user position. The distance between the true and estimated positions was stored as the positioning error. The process resulted in the set of positioning errors corresponding to the estimate used and the topology of the BSs involved. At the final stage the CEP67 and CEP95 were evaluated.

As follows from the results presented in Figure 2-5, the usage of incircle center as the position estimate allows to get better accuracy than the usage of centroid or orthocenter. The accuracy of such an approach is close to the accuracy obtained with the maximum likelihood method. If the mobile is located by $\mathrm{BS}_{1}, \mathrm{BS}_{2}$ and $\mathrm{BS}_{3}$ the accuracy decreases by only 5-6\% compared to the performance of the maximum likelihood estimate. This deterioration has weak dependency upon the value of bearing error. For the second scenario, where the mobile is located by $\mathrm{BS}_{1}, \mathrm{BS}_{2}$ and $\mathrm{BS}_{4}$ the accuracy decreases by $15-20 \%$ against the accuracy of maximum likelihood approach. The accuracy dependence on the value of bearing error is stronger now.

The actual requirements on location accuracy can be found, for example, in [2]. For network-based technologies it claims to require accuracy of 100 meters for $67 \%$ of all calls and an accuracy level of 300 meters for $95 \%$ of all calls. Therefore, the requirements are $C E P 67=100$ $\mathrm{m}$ and $C E P 95=300 \mathrm{~m}$. Under the assumption of $d=1000$ $\mathrm{m}$ (this value of BS-to-BS distance is usual for an urban

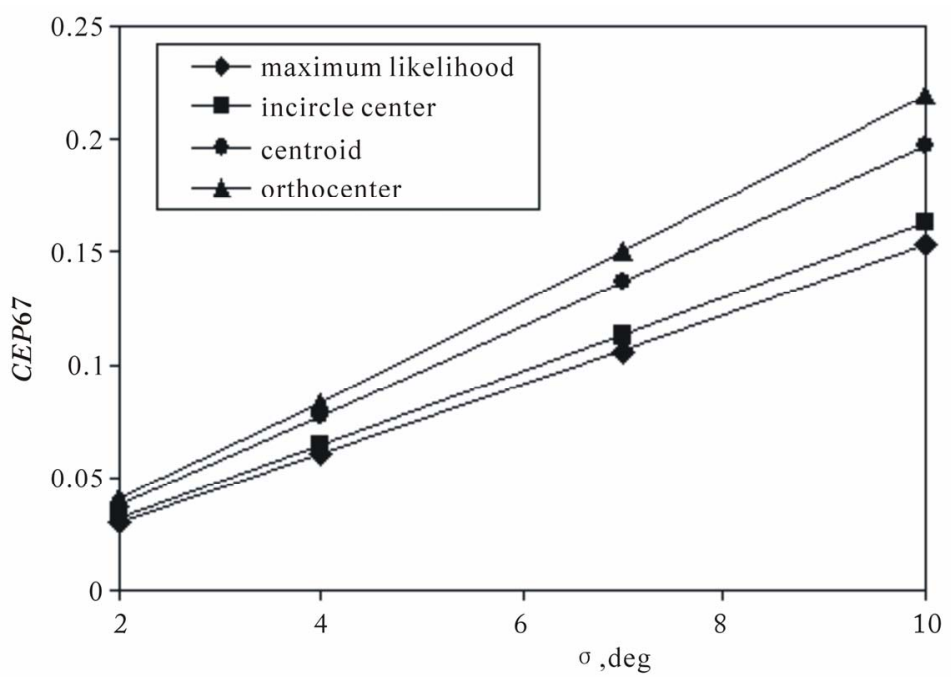

Figure 2. CEP67 against standard deviation of bearing error for scenario 1. 


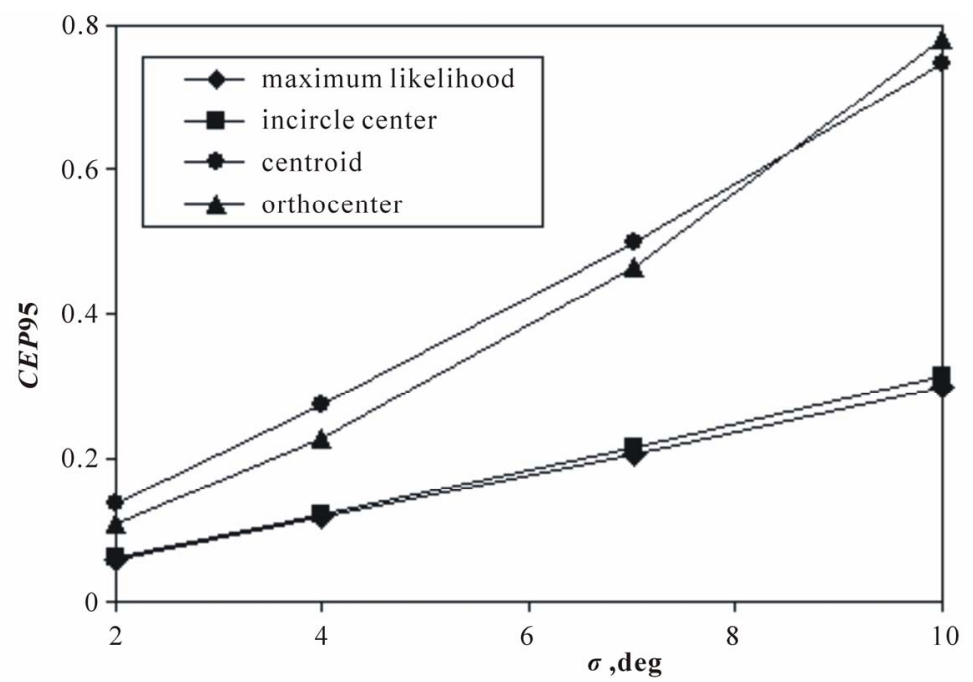

Figure 3. CEP95 against standard deviation of bearing error for scenario 1.

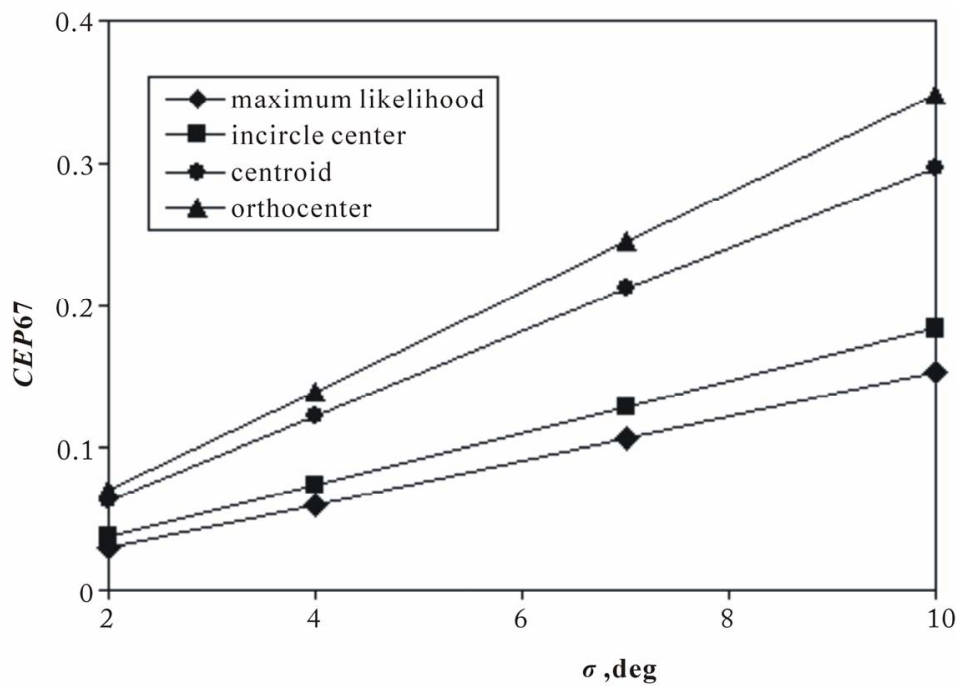

Figure 4. CEP67 against standard deviation of bearing error for scenario 2.

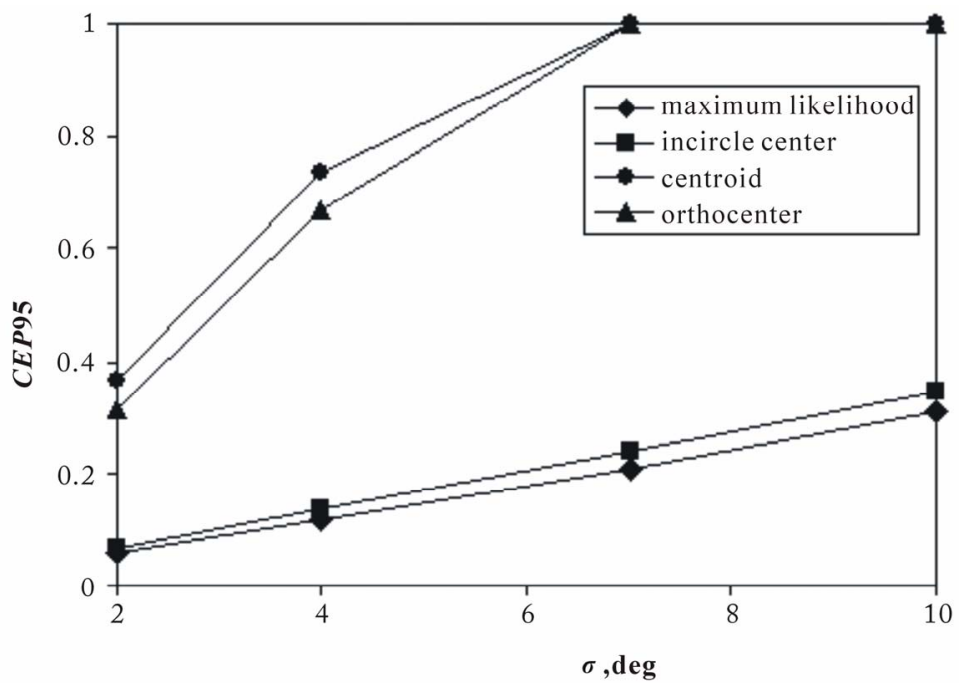

Figure 5. CEP95 against standard deviation of bearing error for scenario 2. 
environment) and the usage of the incircle center as the estimate, the location accuracy for the first scenario sat isfies the requirement as long as $\sigma \leq 6^{\circ}$ (for CEP67) and $\sigma \leq 9^{\circ}$ (for CEP95). Considering the second scenario, it is easy to see that the corresponding limit values are $\sigma \leq$ $5^{\circ}$ (for CEP67) and $\sigma \leq 8^{\circ}$ (for CEP95). It should be noted that for a smaller BS-to-BS distance, larger bearing errors are acceptable.

The presented results also show that the use of centroid or orthocenter as the position estimates leads to the greater positioning errors (compared to the incircle center). As follows from comparison of the curves, the accuracy deterioration in terms of the CEP95 is greater than in terms of the CEP67. This means that corresponding CDFs appear to have longer tails due to rare but significant positioning errors.

\section{Conclusions}

An alternative approach to the maximum likelihood one has been proposed. It involves the estimation of the mobile's position by a closed-form analytical expression. The obtained results have demonstrated that the estimate coinciding with the intersection point of the bearing triangle's angle bisectors (the so-called 'incircle centre') has an accuracy similar to the accuracy of the maximum likelihood estimate. Taking into account the simplicity of its evaluation, the 'incircle centre' estimate may be of interest from a practical point of view.

Acknowledgments: The authors would like to thank D.D.N.Bevan for support and collaboration.

\section{References}

[1] J. Edwards, "TELECOSMOS: The next great telecom revolution,” John Wiley \& Sons, New York, 2005.

[2] FCC Docket No. 94-102, "In the Matter of Revision of the Commission's Rules to Ensure Compatibility with Enhanced 911 Emergency Calling Systems,” Third report and order, FCC 99-245, 1999.

[3] O. Besson, F. Vincent, P. Stoica, and A. B. Gershman, "Approximate Maximum Likelihood Estimators for Array Processing in Multiplicative Noise Environments," IEEE Transactions on Signal Processing, Vol. 48, No. 9, 2000, pp. 2506-2518.

[4] S. Valaee, B. Champagne and P. Kabal, "Parametric Localization of Distributed Sources,” IEEE Transactions on Signal Processing, Vol. 43, No. 9, 1995, pp. 2144-2153.

[5] V. T. Ermolayev, A. G. Flaksman, D. D. N. Bevan and I. M. Averin, "Estimation of the Mobile User Position in the Cellular Communication System in a Multipath Environment of Signal Propagation," Radiophysics and Quantum Electronics, Vol. 51, No. 2, 2008, pp. 145-152.

[6] D. D. N. Bevan, V. T. Ermolayev, A. G. Flaksman and I. M. Averin, "Gaussian Channel Model for Mobile Multipath Environment,” EURASIP Journal on Applied Signal Processing, Vol. 2004, No. 9, 2004, pp. 1321-1329.

[7] http://en.wikipedia.org/wiki/Triangle 